\title{
Are time-trends of smoking among pregnant immigrant women in Sweden determined by cultural or socioeconomic factors?
}

\author{
Kontie M Moussa*1, P-O Ostergren ${ }^{\dagger 1}$, Frida Eek ${ }^{+2}$ and Anton E Kunst ${ }^{+3}$
}

\begin{abstract}
Background: The widening socioeconomic gap in smoking during pregnancy remains a challenge to the Swedish antenatal care services. However, the influence of cultural factors in explaining the socioeconomic differences in smoking during pregnancy is not clear among the immigrant women. The aim of this study was to investigate whether the development of smoking prevalence among pregnant immigrant women in Sweden followed the trajectory which could be expected from the stages of the global smoking epidemic model in the women's countries of origin, or not.

Methods: Delivery data on pregnancies in Sweden from 1982 to 2001 was collected from the Swedish Medical Birth Registry. From a total of 2,224,469 pregnant women during this period, all immigrant pregnant women ( $n=234,731)$ were selected to this study. A logistic regression analysis and attributable fraction were used to investigate the association between smoking during pregnancy and the socioeconomic differences among immigrant women.

Results: Overall, the prevalence of smoking among pregnant immigrant women decreased from $30.3 \%$ in 1982 to $11.0 \%$ in 2001, albeit with remarkable differences between educational levels and country of origin. The greatest decline of absolute prevalence was recorded among low educated women $(27,9 \%)$ and among other Nordic countries $(17,9 \%)$. In relative terms, smoking inequalities increased between educational levels regardless of country of origin. The odds ratios for low educational level for women from other Nordic countries increased from $4.9(95 \% \mathrm{Cl} 4.4-5.4)$ in 1982 to 13.4 (95\% Cl 11.2-16.2) in 2001, as compared to women with high education in the same group. Further, the total attributable fraction for educational difference increased from 55\% in 1982 to $62 \%$ in 2001, demonstrating the strong effect of educational attainment.

Conclusions: Our hypothesis that the socioeconomic time trend of smoking based on the stage of the world wide tobacco epidemic model related to country of origin of the immigrant women was not supported by our analyses. Our findings does not support a call for specific "culture sensitive" antismoking policies or interventions in Sweden or similar countries, but reinforce the existing evidence with a focus on women with a low educational level, regardless of cultural background.
\end{abstract}

\section{Background}

Smoking during pregnancy has been identified as a major risk factor both for future health of the woman and of the fetus/child [1], which gained a considerable public health attention in Sweden. As a result, an intensive antitobacco intervention in antenatal care services were

\footnotetext{
* Correspondence: kontie.moussa@med.lu.se

1 Department of Clinical Sciences Malmö, Division of Social Medicine and Global Health, Lund University, Malmö University Hospital, Malmö, Sweden + Contributed equally

Full list of author information is available at the end of the article
}

launched, which in turn contributed to a significant decline of smoking prevalence among pregnant women. However this decline was more rapid among women with higher socioeconomic status, and therewith widening social inequalities in smoking in Sweden was inevitable [2].

Women with low socioeconomic status include immigrants, lone mothers and young women, who remain as a major challenge to the tobacco interventions in the country. It has been debated whether there is a specific need 
for culturally tailored programmes, in order to reach pregnant immigrants, who now contribute to more than $20 \%$ of yearly deliveries in Sweden.

During the last five decades Sweden has experienced several waves of immigration. The immigration patterns varied from work force migration in the 1960s and 1970s, mostly from other Nordic and other European countries, to a growing proportion of asylum seekers and humanitarian refugees in late 1990s, now also from non-European countries [3]. Stress arising from displacement and introduction into new environment has been shown to expose immigrants to excess health risks and potential health hazards [4]. Consequently, both low socioeconomic- and immigrant status are associated with the risks of less benefit to public health interventions, which in turn contribute to widening health gap, [5-8] and smoking has been identified as an important factor behind these inequalities [2,9].

Longitudinal data on the development over time of the socioeconomic pattern of smoking in pregnant immigrant women is sparse in the scientific literature and the few available studies indicate the presence of behaviour differences in smoking during pregnancy between immigrant and native women in UK [10] and USA [11]. Other studies have reported that immigrant women from low income countries bring with them beliefs, traditions, and cultural practices of their home countries such as negative attitudes towards smoking among women [12]. However, after longer stay in their new countries with greater access to tobacco and fewer normative restrictions, immigrant women may adopt the smoking habits of their host countries $[11,13,14]$. A longitudinal approach that sheds light on the socioeconomic and cultural impact on smoking habits would be of great relevance for understanding the development of health inequalities [1,15-17].

The tendency to respond to health messages during pregnancy may differ due to socio-cultural backgrounds and to the stage of the global tobacco epidemic in the countries of origin of the women. The global tobacco epidemic model was originally based on the progression of cigarette smoking and smoking induced mortality as a general phenomenon and according to the socioeconomic patterning. The model thereby describes different stages of the epidemic [18]. In the first stage of the epidemic, the smoking prevalence increases among men especially in higher socioeconomic strata but remains at low levels among all women. Most of countries in subSaharan Africa fall to this stage. In the second stage, smoking continues to increase among all socioeconomic levels of men reaching a peak and now there is also a rapid increase among women in high socioeconomic position. The concerned countries in this stage are: countries in South East Asia, North Africa, Latin America as well as Japan and China. The third stage of the tobacco epidemic model includes countries in Southern and Eastern Europe, where smoking prevalence is high in both sexes but start to decline rapidly among men in higher socioeconomic strata. Moreover, the prevalence starts to decrease even among women with high education but slowly compared to men. In the fourth phase, smoking decreases in slower fashion among both men and women, however more in men and more in higher socioeconomic strata. Countries in Western Europe, USA, Canada and Australia appear in stage four of the epidemic. During this phase, smoking prevalence will remain high among those in low socioeconomic strata, and thus there is a situation of growing inequalities in this major health determinant [19-21].

Therefore, a comparison between time-trends of smoking among pregnant immigrant women in Sweden and the same trends in the general population may provide useful information for the evaluation of the impact of the Swedish anti-tobacco policies in the two mentioned groups. Since the general trends have been analyzed in a previous study[2], those findings need to be supplemented with a specific analysis regarding the pregnant immigrant women during the same time period.

The aim of this study was to investigate whether the development of the smoking prevalence at the first antenatal visit in pregnant immigrant women in Sweden during the period 1982-2001, and the socioeconomic distribution of smoking in this group, followed the trajectory which could be expected from the stages of the global smoking epidemic model in the women's countries of origin, or not.

\section{Methods \\ Study population}

Data on all pregnancies in Sweden resulting in a delivery from 1982 to 2001 was collected from the Swedish Medical Birth Registry (MBR), which preserves health profiles, marital status, age, nationality, and other demographic factors for all pregnant women in Sweden who participate in the national health system [22]. Our total study cohort consisted of 2,224,469 individuals. Out of these 234,731 women born in other countries than Sweden were targeted for this study. A similar analysis based on the same data source has been described in detail elsewhere [2].

\section{Definitions}

Smoking status at first antenatal care visit was categorised and assessed as follows: 1) Non-smoker, 2) smoke one to nine cigarettes per day, and 3) smoke ten or more cigarettes per day.

Outcome variable: A woman who smoked at least one cigarette per day at the time of her first antenatal visit was classified as a smoker. 


\section{Exposure variables}

Country of origin options were, either born in a) Other Nordic countries (Denmark, Finland, Iceland and Norway), b) Other European countries, or c) Non-European countries.

The education variable was coded into three levels of education, according to number of years a person was enrolled in school, i.e., low educational level (up to nine years), middle educational level (10 to 12 years), and high educational level (more than 12 years).

Marital status: Married women or those women cohabiting with a partner were classified as married; other pregnant women were classified as single mothers.

Age was categorised into three ranges: 16-24 years, 25 to 34 years, and 35-44 years. In our database the age of pregnant women ranged from 16-44 years.

\section{Statistical analyses}

We began by using prevalence as the measure for comparisons over time between the groups studied in order to capture tendencies on an absolute level. Next, multiple logistic regression analyses were performed, yielding odds ratios based on prevalences. In order to eliminate potential confounding from age and marital status, a step-wise multiple logistic regression analysis was also performed, yielding adjusted odds ratios. Prevalences and odds ratios (OR) were calculated using SPSS software Version 16.0 [23].

The attributable fraction (AF) was calculated using the formula: $\mathrm{AF}=(\mathrm{OR}-1) / \mathrm{OR}$, where $\mathrm{OR}$ is the adjusted odds ratio generated by multiple logistic regression analysis [24]. Total attributable fraction (TAF) was calculated as follows:

$$
\mathrm{TAF}=\sum_{\mathrm{i}}(\mathrm{sTAF})=\sum_{\mathrm{i}}\left(\mathrm{AF}_{\mathrm{i}}{ }^{*} \mathrm{P}_{\mathrm{i}}\right)
$$

where $A_{i}$ is the attributable fraction for smoking at first antenatal visit for a specific stratum (here: educational level), and $\mathrm{P}_{\mathrm{i}}$ represents the proportion of all cases that fall in this stratum. The expression within the parenthesis thus represents the stratum-specific total attributable fraction (sTAF), and indicates the summation of all the strata-specific calculations, which in turn results in the overall TAF. For those with the highest level of education, the AF and sTAF are by definition zero [25]. Finally, overall TAF is the summation of the STAFs and represents the proportion of smoking that would not exist if all pregnant women had had the same prevalence as those with highest level of education, under the assumption that there is a causal pathway between educational level and the outcome variable.

\section{Results}

The demographic characteristics of immigrant pregnant women in our study are provided in table 1 . The smoking prevalence during pregnancy in Sweden decreased from $30.3 \%$ in 1982 to $11.0 \%$ in 2001 . Women from other Nordic countries were a great majority $(55.2 \%)$ in 1986 ; however this group shrank to $14.7 \%$ in 2001 . Oppositely, the proportion of women from Non-European countries increased from $18.6 \%$ in 1982 to $56.1 \%$ in 2001 , while the proportion remained constant for women from European countries. During the period studied, the proportion of women with low-, middle- and high educational level was $28 \%, 46 \%$, and $26 \%$, respectively. The large majority of women were in age group of 25-34 years, married or cohabiting with a partner (Table 1 ).

The prevalence of smoking at the time of the first antenatal visit among immigrant women decreased in all educational groups, but more so in the lower and middle categories, as compared with the highest. Subsequently, the smoking prevalence among pregnant women with high educational level has decreased from $16.1 \%$ to $4.6 \%$ (a prevalence difference of 11.5\%), and for women with middle educational level the corresponding decrease was from $31.8 \%$ to $12.1 \%$ (a prevalence difference of $19.7 \%$ ). However, the highest prevalence reduction was recorded by women with low educational level from $44.6 \%$ to $16.7 \%$ (a prevalence difference of $27.9 \%$ ). Likewise, the decline of the prevalence among pregnant women from different countries 1982-2001 shows that this has decreased from $37.9 \%$ to $20.0 \%$ among women from other Nordic countries; from $26.4 \%$ to $16.9 \%$ among women from other European countries; and from $13.3 \%$ to $5.5 \%$ among women from Non-European countries. Thus, on an absolute scale smoking decreased less among women with highest educational level. Among the age groups, the highest absolute reduction in smoking prevalence was seen among women 16-24 years of age followed by those in age group 25-34 years compared to eldest group (24.7\%, $18.8 \%$, and $12.4 \%$, respectively). Moreover, a considerable absolute reduction in smoking prevalence has been recorded among single mothers and women born in other Nordic countries. As a general pattern, the highest prevalence declines were seen in the groups that had the highest smoking rates at baseline 1982 and the sociodemographic disparities seemed to be narrowing on an absolute scale (Table 2).

Table 3 shows the prevalence of smoking at each educational level according to country of origin and the crude odds ratios with $95 \% \mathrm{CI}$. The results demonstrate an increasing relative difference in odds ratios during the period studied between educational levels regardless of country of origin. The crude OR for pregnant women with low educational level from other Nordic countries than Sweden increased from 4.9 (4.4-5.4) in 1982 to 13.4 (11.2-16.2) in 2001, as compared to women with high education in the same group. During the period of 19822001, the crude ORs for smoking during pregnancy among women with low education from other European countries increased from $2.9(2.4-3.6)$ to $4.0(3.5-4.5)$ 
Table 1: Demographic description of pregnant foreign-born (migrant) women at first visit at antenatal clinic from 19822001 and valid percent $(\%)$ presented in 5 years intervals.

\begin{tabular}{|c|c|c|c|c|c|c|c|c|}
\hline & \multicolumn{2}{|c|}{ 1982-1986 } & \multicolumn{2}{|c|}{ 1987-1991 } & \multicolumn{2}{|c|}{ 1992-1996 } & \multicolumn{2}{|c|}{$1997-2001$} \\
\hline & $\mathbf{n}$ & $\%$ & $\mathbf{n}$ & $\%$ & $\mathbf{n}$ & $\%$ & $\mathbf{n}$ & $\%$ \\
\hline Smoking status & & & & & & & & - \\
\hline Smokers & 12081 & 30.3 & 13440 & 25.6 & 9545 & 17.4 & 7133 & 11.0 \\
\hline None-smokers & 27727 & 69.7 & 39118 & 74.4 & 45245 & 82.6 & 57862 & 89.0 \\
\hline Missing & 8842 & -- & 4849 & -- & 3144 & -- & 5745 & -- \\
\hline Total & 48650 & & 57407 & & 57934 & & 70740 & \\
\hline \multicolumn{9}{|l|}{ Education } \\
\hline Low level & 7916 & 28.9 & 12238 & 29.9 & 12431 & 26.0 & 16799 & 27.3 \\
\hline Middle level & 13140 & 48.0 & 19867 & 48.3 & 22560 & 47.1 & 25919 & 42.1 \\
\hline High level & 6320 & 23.1 & 9026 & 21.9 & 12912 & 27.0 & 18858 & 30.6 \\
\hline Missing data & 21274 & & 16276 & & 10031 & & 9164 & \\
\hline Total & 48650 & & 57407 & & 57934 & & 70740 & \\
\hline \multicolumn{9}{|l|}{ Age group } \\
\hline $16-24$ years & 13349 & 27.4 & 13858 & 24.1 & 11901 & 20.5 & 14849 & 21.0 \\
\hline $25-34$ years & 28701 & 59.0 & 34784 & 60.6 & 35931 & 62.0 & 42621 & 60.3 \\
\hline 35-years & 6600 & 13.6 & 8765 & 15.3 & 10102 & 17.4 & 13270 & 18.8 \\
\hline Missing data & 0 & & 0 & & 0 & & 0 & \\
\hline Total & 48650 & & 57407 & & 57934 & & 70740 & \\
\hline \multicolumn{9}{|l|}{ Marital status } \\
\hline Married & 39507 & 95.9 & 50208 & 95.4 & 49179 & 92.6 & 62024 & 94.5 \\
\hline Single & 1697 & 4.1 & 2408 & 4.6 & 3929 & 7.4 & 2580 & 5.5 \\
\hline Missing data & 7446 & & 4791 & & 4826 & & 5136 & \\
\hline Total & 48650 & & 57407 & & 57934 & & 70740 & \\
\hline \multicolumn{9}{|l|}{ Country of origin } \\
\hline Nordic countries & 26864 & 55.2 & 26065 & 45.4 & 18310 & 31.6 & 10383 & 14.7 \\
\hline European countries & 12747 & 26.2 & 15598 & 27.2 & 16123 & 27.8 & 20700 & 29.3 \\
\hline Non-European countries & 9039 & 18.6 & 15744 & 27.4 & 23501 & 40.6 & 39657 & 56.1 \\
\hline Missing data & 0 & & 0 & & 0 & & 0 & \\
\hline Total & 48650 & & 57407 & & 57934 & & 70740 & \\
\hline
\end{tabular}

$(n=234731)$

compared to their counterparts with high level of education. Furthermore, the crude ORs increased from 1.2 (1.6-2.8) to 3.4 (3.0-4.0) for women with low educational level from Non-European compared to those with high educational level (Table3). When adjusted for age and marital status the odds ratios between educational groups changed marginally (data not shown).

Table 4 shows the association between smoking at first antenatal visit and educational level, adjusted OR (for age marital status, and country of origin), AF, sTAF, and overall TAF between 1982 and 2001. A further illustration shows that STAF for low educated women increased from $29 \%$ to $33 \%$ between the periods 1982 to 2001 , indicating the widening gap in smoking between educational levels among immigrant women at the time of their first antenatal visit. Further, the total attributable fraction for educational differences increased from $55 \%$ in 1982 to $62 \%$ in 2001, demonstrating that the association with educational attainment regarding the studied type of smoking behaviour, increased somewhat during the studied period. For instance, the calculation of attributable fraction was demonstrated according to table 4. Attributable fraction $(\mathrm{AF})=(\mathrm{OR}-1) / \mathrm{OR}$; AF for low educational level $1982-1986$ would be $(3.6-1 / 3.6=0.72)$, hence stratum specific total attributable fraction (sTAF) for that group can be calculated by multiplying the proportion of the 
Table 2: Number of pregnant immigrant smokers ( $\mathrm{n}$ ) and smoking prevalence (\%) according to socio-demographic variables during first visit at antenatal clinic from 1982-2001 presented in 5 years intervals

\begin{tabular}{|c|c|c|c|c|c|c|c|c|}
\hline \multirow[b]{2}{*}{ Educational level } & \multicolumn{2}{|c|}{$1982-86$} & \multicolumn{2}{|c|}{ 1987-1991 } & \multicolumn{2}{|c|}{ 1992-1996 } & \multicolumn{2}{|c|}{ 1997-2001 } \\
\hline & $\mathbf{n}$ & $\%$ & $\mathbf{n}$ & $\%$ & $\mathbf{n}$ & $\%$ & $\mathbf{n}$ & $\%$ \\
\hline High level & 823 & 16.1 & 1044 & 12.5 & 1019 & 8.3 & 797 & 4.6 \\
\hline Middle level & 3461 & 31.8 & 5076 & 27.8 & 4269 & 19.9 & 2889 & 12.1 \\
\hline Low level & 2944 & 44.6 & 3905 & 35.1 & 3052 & 26.5 & 2587 & 16.7 \\
\hline Total & 7228 & & 10025 & & 8340 & & 6273 & \\
\hline \multicolumn{9}{|l|}{ Age group } \\
\hline $16-24$ years & 4022 & 37.0 & 3800 & 30.1 & 2092 & 18.6 & 1687 & 12.3 \\
\hline $25-34$ years & 6725 & 28.7 & 7713 & 24.2 & 5672 & 16.7 & 4002 & 10.2 \\
\hline 35-years & 1334 & 24.4 & 1927 & 24.0 & 1781 & 18.7 & 1444 & 12.0 \\
\hline Total & 12081 & & 13440 & & 9545 & & 7133 & \\
\hline \multicolumn{9}{|l|}{ Marital status } \\
\hline Married & 10968 & 29.3 & 11569 & 24.4 & 7625 & 16.0 & 6176 & 10.3 \\
\hline Single & 868 & 54.2 & 1113 & 48.9 & 1324 & 35.0 & 749 & 21.7 \\
\hline Total & 11836 & & 12682 & & 8949 & & 6925 & \\
\hline \multicolumn{9}{|l|}{ Country of origin } \\
\hline Other Nordic countries & 8392 & 37.9 & 8389 & 35.0 & 5005 & 28.8 & 1903 & 20.0 \\
\hline Other European countries & 2703 & 26.4 & 3447 & 24.2 & 2791 & 18.4 & 3252 & 16.9 \\
\hline Non-European countries & 986 & 13.3 & 1604 & 11.2 & 1749 & 7.9 & 1978 & 5.5 \\
\hline Total & 12081 & & 13440 & & 9545 & & 7133 & \\
\hline
\end{tabular}

group in that stratum by AF that yields; $0.407^{*} 0.72=0.29$ or $29 \%$ (Table 4 ).

Figure 1 illustrates trends in smoking prevalence among immigrant pregnant women in Sweden according to country of origin from 1982-2001. The overall trend shows that smoking during pregnancy has declined in all groups. However, the highest absolute reduction in prevalence was seen among women born in other Nordic countries with $18.0 \%$, which also had highest prevalence of smoking 1982 compared to other groups. The gap of smoking prevalence between women born in Nordic and European countries is narrowing, however still remaining higher compared to women born in non-European countries (Figure 1).

\section{Discussion}

The main findings of our study indicate that there has been a noteworthy overall decline in smoking prevalence among pregnant immigrant women in Sweden during the period studied. In absolute terms the smoking prevalence declined among all groups indicating reduced socioeconomic inequality. However, a relative increase of inequality was shown which persisted also after adjusting for age and marital status. The increasing socioeconomic differences were more evident among women born in Nordic countries. Among this group the adjusted ORs for low educational level increased from 4.9 (4.4-5.4) to 13.4 (11.2-16.2) during 1982-2001. The corresponding increase among women from non-European countries during the same period was from OR 2.1 (1.6-2.8) to 3.4 (3.0-4.0). The widening socioeconomic gap in smoking during pregnancy among women from other Nordic countries has been shown to be similar to the trend observed among Swedish pregnant women [2]. Our results show that the socioeconomic inequality in smoking widened between women born in Europe and women born in non-European countries. The observations do not support the assumption that smoking trends in the studied groups of immigrant women could be predicted by the world-wide tobacco epidemic model, i.e. by comparing with the socioeconomic trends in smoking among women in their particular countries of origin. According to this model, women with high education from nonEuropean countries would be expected to have higher smoking prevalence compared to women from Nordic countries. In contrast, our findings show that women with high education smoke less during pregnancy, regardless of the stage of the tobacco epidemic of their countries of origin.

Thus, our original hypothesis must be rejected on basis of our findings and alternative explanations should be sought. 
Table 3: Prevalence (\%) of smoking during pregnancy at first antenatal care visit and crude odds ratios (OR) with $95 \%$ confidence intervals (CIs) for each five-year period according to educational levels and country of origin from $1982-2001$.

\begin{tabular}{|c|c|c|c|c|c|c|}
\hline & \multicolumn{2}{|c|}{ Other Nordic Countries } & \multicolumn{2}{|c|}{ Other European Countries (Nordic countries excluded) } & \multicolumn{2}{|c|}{$\begin{array}{l}\text { Non-Europeans } \\
\text { Countries }\end{array}$} \\
\hline & $\%$ & OR $(95 \% \mathrm{CI})$ & $\%$ & OR $(95 \% \mathrm{Cl})$ & $\%$ & OR $(95 \% \mathrm{Cl})$ \\
\hline \multicolumn{7}{|l|}{ 1982-1986 } \\
\hline High level & 16.9 & 1.0 & 16.6 & 1.0 & 12.6 & 1.0 \\
\hline Middle level & 36.1 & $2.8(2.5-3.1)$ & 27.8 & $1.9(1.6-2.3)$ & 16.4 & $1.4(1.1-1.8)$ \\
\hline Low level & 49.7 & $4.9(4.4-5.4)$ & 36.9 & $2.9(2.4-3.6)$ & 23.4 & $2.1(1.6-2.8)$ \\
\hline \multicolumn{7}{|l|}{ 1987-1991 } \\
\hline High level & 14.0 & 1.0 & 13.8 & 1.0 & 7.5 & 1.0 \\
\hline Middle level & 33.9 & $3.2(2.9-3.5)$ & 25.1 & $2.1(1.8-2.4)$ & 13.4 & $1.9(1.6-2.3)$ \\
\hline Low level & 51.4 & $6.5(5.9-7.2)$ & 33.4 & $3.1(2.7-3.7)$ & 13.2 & $2.0(1.6-3.7)$ \\
\hline \multicolumn{7}{|l|}{ 1992-1996 } \\
\hline High level & 10.5 & 1.0 & 10.5 & 1.0 & 4.8 & 1.0 \\
\hline Middle level & 28.6 & $3.4(3.0-3.8)$ & 19.1 & $2.0(1.8-2.3)$ & 9.8 & $2.1(1.8-2.5)$ \\
\hline Low level & 49.6 & $8.4(7.4-9.4)$ & 25.4 & $2.9(2.5-3.3)$ & 10.3 & $2.3(1.9-2.7)$ \\
\hline \multicolumn{7}{|l|}{$1997-2001$} \\
\hline High level & 5.6 & 1.0 & 7.8 & 1.0 & 2.5 & 1.0 \\
\hline Middle level & 20.6 & $4.4(3.7-5.2)$ & 17.6 & $2.5(2.2-2.8)$ & 5.9 & $2.5(2.1-2.9)$ \\
\hline Low level & 44.4 & $\begin{array}{c}13.4(11.2- \\
16.2)\end{array}$ & 25.2 & $4.0(3.5-4.5)$ & 8.1 & $3.4(3.0-4.0)$ \\
\hline
\end{tabular}

When considering the development of smoking patters in general in Sweden as well as the socioeconomic pattern of smoking of immigrants, it could be important to consider the process of acculturation. Berry's typology of acculturation strategies is based on the combination of two main factors, preservation of the culture of origin and degree of participation in the new country [26]. This will yield four situations of the immigrant population, marginalization (low degree of keeping the original culture and low degree of participation in the new society), isolation (high degree of keeping the original culture and low degree of participation), assimilation (low degree of keeping original culture and high degree of participation) and finally integration (high degree of keeping original culture and high degree of participation). Interpreting the development of smoking prevalence among pregnant immigrant women according to Berry's model indicate that our findings fit less with aspects of marginalization and isolation, but better with Berry's description of assimilation and integration. Hence, the trend of smoking habits among immigrant women seems to be shaped by the same influences as those which have affected the whole population, (e.g. general Swedish tobacco prevention initiatives on women's smoking). Thus, our findings strongly imply that the most important determinant of smoking trend among pregnant immigrant women in Sweden was associated with the level of education rather than women's cultural background.

An important factor to be taken into consideration, is the change over time regarding the geographic region of the country of origin of the immigrant women, namely from Europe (particularly other Nordic countries) in the beginning of the studied period, to a growing proportion of immigrants from non-European countries. In those countries smoking prevalence is usually low among all women, regardless of educational level, which ought to favour less socioeconomic inequality over time if they retained their original smoking habits, if the original hypothesis were true. This is also contrary to our analyses, since inequality did not decrease over time. This picture is also more compatible with our alternative hypothesis based on Berry's typologies of integration.

A third factor that might be considered is the role that 'healthy immigrant effect' plays in immigrants' health behaviour. The selection of healthy individuals in the migration process might contribute to that non-smokers actively would make a healthier choices to not initiate smoking, assuming that they originate from a society 
Table 4: Number of foreign-born smokers ( $n$ ) during pregnancy and their proportion (\%) in each educational level stratum, adjusted Odds Ratios (aOR)* and 95\% Confidence Interval (CI) for smoking in those strata, and Attributable Fraction (AF), Stratum-specific Total Attributable Fraction (sTAF), and overall Total Attributable Fraction (TAF) for each five-year period.

\begin{tabular}{|c|c|c|c|c|c|c|}
\hline Educational levels & $\mathbf{n}$ & $\%$ & $\mathrm{aOR}^{*}(95 \% \mathrm{Cl})$ & AF & STAF & TAF \\
\hline \multicolumn{7}{|l|}{ 1982-1986 } \\
\hline High educational level & 823 & 11.4 & 1.0 & 0 (Ref) & & \\
\hline Middle educational level & 3461 & 47.9 & $2.2(2.0-2.4)$ & 0.55 & 0.26 & \\
\hline Low educational level & 2944 & 40.7 & $3.6(3.3-3.9)$ & 0.72 & 0.29 & \\
\hline TAF, all levels of education & & & & & & 0.55 \\
\hline \multicolumn{7}{|l|}{ 1987-1991 } \\
\hline High educational level & 1044 & 10.4 & 1.0 & 0 (Ref) & & \\
\hline Middle educational level & 5076 & 50.6 & $2.6(2.4-2.8)$ & 0.62 & 0.31 & \\
\hline Low educational level & 3905 & 39.0 & $4.3(3.9-4.7)$ & 0.77 & 0.30 & \\
\hline TAF, all levels of education & & & & & & 0.61 \\
\hline \multicolumn{7}{|l|}{ 1992-1996 } \\
\hline High educational level & 1019 & 12.2 & 1.0 & 0 (Ref) & & \\
\hline Middle educational level & 4269 & 51.2 & $2.5(2.3-2.7)$ & 0.60 & 0.31 & \\
\hline Low educational level & 3052 & 36.6 & $4.1(3.8-4.5)$ & 0.76 & 0.28 & \\
\hline TAF, all levels of education & & & & & & 0.59 \\
\hline \multicolumn{7}{|l|}{$1997-2001$} \\
\hline High educational level & 797 & 12.7 & 1.0 & 0 (Ref) & & \\
\hline Middle educational level & 2889 & 46.1 & $2.8(2.6-3.1)$ & 0.64 & 0.29 & \\
\hline Low educational level & 2587 & 42.2 & $4.8(4.4-5.3)$ & 0.79 & 0.33 & \\
\hline TAF, all levels of education & & & & & & 0.62 \\
\hline Total smokers 1982-2001 & 31866 & & & & & \\
\hline
\end{tabular}

* adjusted for age and marital status.

$(\mathrm{n}=31866)$

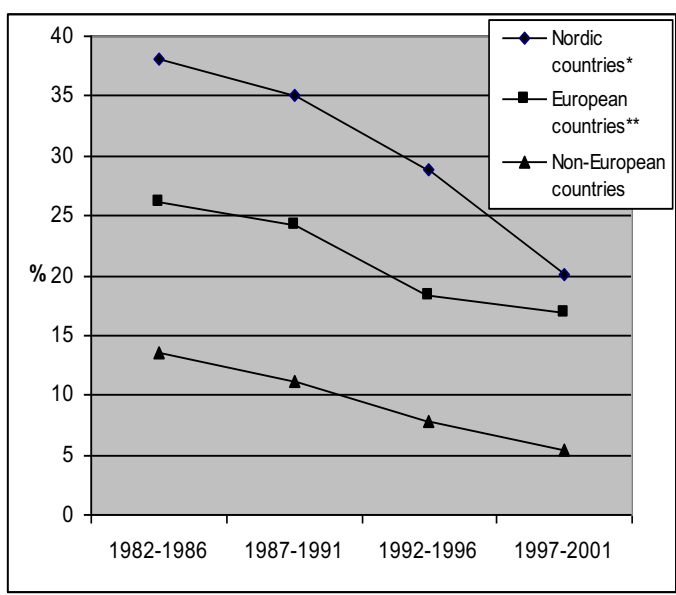

* Sweden excluded

** Nordic countries excluded

Figure 1 Smoking prevalence during pregnancy among immigrant women in Sweden 1982-2001. with lower tobacco consumption, where cultural and traditional values have a protective impact [27], in combination with Swedish tobacco control policy.

Strengths of this study are the size of the sample, the long study period, and its limitation to the first generation of immigrant women. Another strength is that data from the Swedish Birth Register is little sensitive to selection bias, since almost all deliveries are taking place in hospitals and are thereby registered.

Under-reporting of the outcome (smoking during first antenatal visit) has been a concern of validity with regard to self reported smoking. Several studies however suggest that self-reported data on smoking to be a good and reliable indicator of the daily smoking $[28,29]$.

The limitations of this study include the difficulty to ascertain the evidence whether the women started or quit smoking in Sweden or in their countries of origin as well as the difficulty to interpret the concept of acculturation since we lack data on numbers of years lived in Sweden and age of immigration. Additionally, our data lacks more 
explanatory variables such as employment status and income.

\section{Conclusions}

Our hypothesis that the socioeconomic time trend of smoking at first ante-natal visit based on the stage of the world wide tobacco epidemic model of the country of origin of the immigrant women was not supported by our analyses. An alternative hypothesis might be based on acculturation strategies, which also ought to depend strongly on integration policies for immigrants in a particular country. Our findings does not support a call for specific "culture sensitive" antismoking policies or interventions in Sweden or similar countries, but reinforce the existing evidence that a focus should be set on women with a low educational level, regardless of cultural background.

\section{Competing interests}

The authors declare that they have no competing interests.

\section{Authors' contributions}

KM led the writing and completed the analyses. P-OO, FE and AK helped in conceiving the study, conceptualising ideas, interpreting the results, and reviewing drafts of the article. All authors read and approved the final manuscript.

\section{Acknowledgements}

This study was made possible by grants from the Swedish Council for Working Life and Social Research, grant number FAS 2005-1121, an ALF grant from the Medical Faculty of Lund University and 'Tackling Socioeconomic Inequalities in Smoking' project funded by European Commission, Public Health Directorate, through the ENSP.

\section{Disclaimer}

Not mentioned

\section{Author Details}

'Department of Clinical Sciences Malmö, Division of Social Medicine and Global Health, Lund University, Malmö University Hospital, Malmö, Sweden, 2Division of Occupational and Environmental Medicine, Lund University, Lund University Hospital, Lund, Sweden and 'BDepartment of Public Health, Academic Medical Centre (AMC), University of Amsterdam, the Netherlands

Received: 21 October 2009 Accepted: 26 June 2010

Published: 26 June 2010

\section{References}

1. Cnattingius S: The epidemiology of smoking during pregnancy: smoking prevalence, maternal characteristics, and pregnancy outcomes. Nicotine Tob Res 2004, 6(Suppl 2):S125-140.

2. Moussa KM, Ostergren PO, Grahn M, Kunst AE, Eek F, Essen B: Socioeconomic differences in smoking trends among pregnant women at first antenatal visit in Sweden 1982-2001: Increasing importance of educational level for the total burden of smoking. Tob Control 2009, 18(2):92-97.

3. Statistics Sweden: Demographic reports 2008:4, Immigrants' migration patterns. Statistics Sweden 2008

4. World Health Organization: Health of migrants - migration flows and the globalized world. 2007 [http://www.who.int/hac/techguidance/ health of migrants/B122 11-en.pdf]. Geneva: WHO Executive board 122nd session Accessed 16 April, 2010

5. Sundquist J, Johansson SE: [Somatic health is poorer among foreignborn than native Swedes]. Lakartidningen 1998, 95(10):1000-1002. 10051006

6. Sundquist J, Johansson SE: The influence of socioeconomic status, ethnicity and lifestyle on body mass index in a longitudinal study. Int J Epidemiol 1998, 27(1):57-63.
7. Gadd M, Johansson SE, Sundquist J, Wandell P: The trend of cardiovascular disease in immigrants in Sweden. Eur J Epidemiol 2005, 20(9):755-760

8. Gadd M, Sundquist J, Johansson SE, Wandell P: Do immigrants have an increased prevalence of unhealthy behaviours and risk factors for coronary heart disease? Eur J Cardiovasc Prev Rehabil 2005, 12(6):535-541.

9. Mackenbach JP, Stirbu I, Roskam AJ, Schaap MM, Menvielle G, Leinsalu M, Kunst AE: Socioeconomic inequalities in health in 22 European countries. N Engl J Med 2008, 358(23):2468-2481.

10. Hawkins SS, Lamb K, Cole TJ, Law C: Influence of moving to the UK on maternal health behaviours: prospective cohort study. Bmj 2008, 336(7652):1052-1055.

11. Perreira KM, Cortes KE: Race/ethnicity and nativity differences in alcohol and tobacco use during pregnancy. Am J Public Health 2006, 96(9):1629-1636.

12. Nierkens V, de Vries H, Stronks K: Smoking in immigrants: do socioeconomic gradients follow the pattern expected from the tobacco epidemic? Tob Control 2006, 15(5):385-391.

13. Harley K, Eskenazi B: Time in the United States, social support and health behaviors during pregnancy among women of Mexican descent. Soc SciMed 2006, 62(12):3048-3061.

14. Celi AC, Rich-Edwards JW, Richardson MK, Kleinman KP, Gillman MW Immigration, race/ethnicity, and social and economic factors as predictors of breastfeeding initiation. Arch Pediatr Adolesc Med 2005 159(3):255-260

15. Cnattingius S, Lambe M: Trends in smoking and overweight during pregnancy: prevalence, risks of pregnancy complications, and adverse pregnancy outcomes. Semin Perinatol 2002, 26(4):286-295.

16. Hogberg $L$, Cnattingius $S$ : The influence of maternal smoking habits on the risk of subsequent stillbirth: is there a causal relation? Bjog 2007, 114(6):699-704

17. Kallen $\mathrm{K}$ : The impact of maternal smoking during pregnancy on delivery outcome. Eur J Public Health 2001, 11(3):329-333.

18. Lopez ADCN, Tapani P: A descriptive model of the cigarette epidemic in developed countries. Tob Control 1994, 3:242-247.

19. Folkhälsorapport 2009: [Public Health Report, 2009]. Stockholm: Socialstyrelsen; 2009.

20. Alberg AJ, Nonemaker J: Who is at high risk for lung cancer? Populationlevel and individual-level perspectives. Semin Respir Crit Care Med 2008, 29(3):223-232

21. Schneider S, Maul H, Freerksen N, Potschke-Langer M: Who smokes during pregnancy? An analysis of the German Perinatal Quality Survey 2005. Public Health 2008, 122(11):1210-1216.

22. National Board of Health and Welfare: Validation of Swedish Medical Birth Register (In Swedish). 2002. 2002-112-4

23. SPSS inc: SPSS Base for windows 16.0 User's Guide. 2007.

24. Greenland S, Drescher K: Maximum likelihood estimation of the attributable fraction from logistic models. Biometrics 1993, 49(3):865-872

25. Rothman K: Epidemiology: an introduction. New York: Oxford University Press inc; 2002.

26. Berry JW: Immigration, Acculturation, and Adaptation. Applied Psychology: An international review 1997, 46(1):5-68.

27. Singh GK, Yu SM: Adverse pregnancy outcomes: differences between US- and foreign-born women in major US racial and ethnic groups. Am J Public Health 1996, 86(6):837-843.

28. Graham H, Owen L: Are there socioeconomic differentials in underreporting of smoking in pregnancy? Tob Control 2003, 12(4):434

29. Vartiainen E, Seppala T, Lillsunde P, Puska P: Validation of self reported smoking by serum cotinine measurement in a community-based study. J Epidemiol Community Health 2002, 56(3):167-170.

\section{Pre-publication history}

The pre-publication history for this paper can be accessed here: http://www.biomedcentral.com/1471-2458/10/374/prepub

doi: $10.1186 / 1471-2458-10-374$

Cite this article as: Moussa et al., Are time-trends of smoking among pregnant immigrant women in Sweden determined by cultural or socioeconomic factors? BMC Public Health 2010, 10:374 